\title{
Left main coronary artery compression in pulmonary arterial hypertension
}

\author{
Kadhem Albadri, Jesper M. Jensen, Evald H. Christiansen, Søren Mellemkjær, Jens Erik Nielsen-Kudsk \\ Department of Cardiology, Aarhus University Hospital, Aarhus, Denmark
}

\begin{abstract}
In patients with pulmonary arterial hypertension (PAH), chest pain is most likely due to right ventricular demand ischemia. We report a patient with idiopathic PAH who developed severe angina due to extrinsic compression of the left main coronary artery (LMCA) from a dilated pulmonary artery trunk. The diagnosis was verified by electrocardiogram after exercise, coronary angiography including intravascular ultrasound, and cardiac multidetector computed tomography (MDCT). The origin of the LMCA was high in the left coronary sinus, facilitating extrinsic compression. The patient was successfully treated by percutaneous coronary intervention with stent implantation in the LMCA. Extrinsic compression of the LMCA is a severe and potentially fatal complication that should be considered in all patients with $\mathrm{PAH}$ and angina. MDCT is the method of choice for first-line diagnosis.
\end{abstract}

Keywords: pulmonary hypertension, chest pain, left main coronary artery.

Pulm Circ 2015;5(4):734-736. DOI: 10.1086/683690.

\section{CASE DESCRIPTION}

Idiopathic pulmonary arterial hypertension (IPAH) was diagnosed in a 49-year-old woman 6 years ago. She was classified as World Health Organization (WHO) functional class (FC) III at the time of diagnosis, but during treatment with an endothelin receptor antagonist (ambrisentan), she improved considerably and became stable in WHO FC II, with a 6-minute walk test distance of $\geq 400 \mathrm{~m}$. She was seen at regular intervals of 4-6 months in our outpatient pulmonary hypertension $(\mathrm{PH})$ clinic. During the latest visit, the patient reported increasing dyspnea and tightening chest pain during physical activity. She had no family history or other risk factors for coronary artery disease. On suspicion of worsening PAH, she was admitted to our PH ward for further evaluation.

Physical examination revealed a normal blood pressure of $110 / 80 \mathrm{mmHg}$, heart rate of 80 beats/minute, and no sign of right- or left-sided heart failure. Transthoracic echocardiography revealed a moderately dilated right ventricle (RV) with an estimated systolic RV pressure of $100 \mathrm{mmHg}$ and a tricuspid annular plane systolic excursion of $1.3 \mathrm{~cm}$. The left ventricle (LV) was small (end-diastolic diameter: $2.8 \mathrm{~cm}$ ) with a mildly depressed LV systolic function (LV ejection fraction: 50\%). The main pulmonary artery (PA) was severely dilated (diameter: $42 \mathrm{~mm}$ ) and later was visualized to be immediately adjacent to the takeoff of the left main coronary artery (LMCA). During a 6-minute walk test, the patient developed severe tightness around the chest after a 5-m walk and was unable to complete the test. An acute 12-lead electrocardiogram (ECG) showed ST segment depression in the inferior and the anterolateral leads compared with the resting 12-lead ECG (Fig. 1).
Coronary arteriography was performed from the right femoral artery and demonstrated a severe ostial LMCA stenosis (Fig. 2). The LMCA originated from a high position in the left sinus of the aortic root. There were no other atherosclerotic changes or stenosis of the coronary arteries. Intravascular ultrasound (IVUS) showed that the ostium of the LMCA was narrowed to a slit without evidence of underlying atherosclerosis. Cardiac computed tomography (ECGgated multidetector computed tomography [MDCT]) confirmed a relatively high origin of the LMCA and a slit-like compression of the ostial part of the LMCA caused by a severely dilated PA trunk (Fig. 3).

The patient underwent uncomplicated percutaneous coronary intervention (PCI) with implantation of a drug-eluting stent in the LMCA. Her symptoms completely resolved after the PCI. She performed a 6-minute walk test with a distance of $390 \mathrm{~m} 1$ day after the PCI and did not experience any chest pain. Right-sided catheterization 1 day before discharge from the hospital confirmed the severity of the patient IPAH as diagnosed in 2008. It showed an atrial pressure of $5 \mathrm{mmHg}$, pulmonary capillary wedge pressure of $12 \mathrm{mmHg}$, mean pulmonary pressure of $58 \mathrm{mmHg}$, cardiac output of $3.8 \mathrm{~L} /$ minute, cardiac index of $1.95 \mathrm{~L} /$ minute $/ \mathrm{m}^{2}$, and pulmonary vascular resistance of 15 Wood units.

\section{DISCUSSION}

Chest pain is one of the common manifestations in patients with $\mathrm{PH} .{ }^{1}$ The well-known explanation for chest pain in $\mathrm{PH}$ is RV hypertrophy and strain leading to demand ischemia. ${ }^{2}$ Therefore, the de-

Address correspondence to Dr. Kadhem Albadri, Department of Cardiology, Aarhus University Hospital, Palle Juul-Jensens Boulevard 99, 8200 Aarhus N, Denmark. E-mail: kadhalba@rm.dk.

Submitted February 9, 2015; Accepted May 13, 2015; Electronically published October 21, 2015.

(C) 2015 by the Pulmonary Vascular Research Institute. All rights reserved. 2045-8932/2015/0504-0017. \$15.00. 

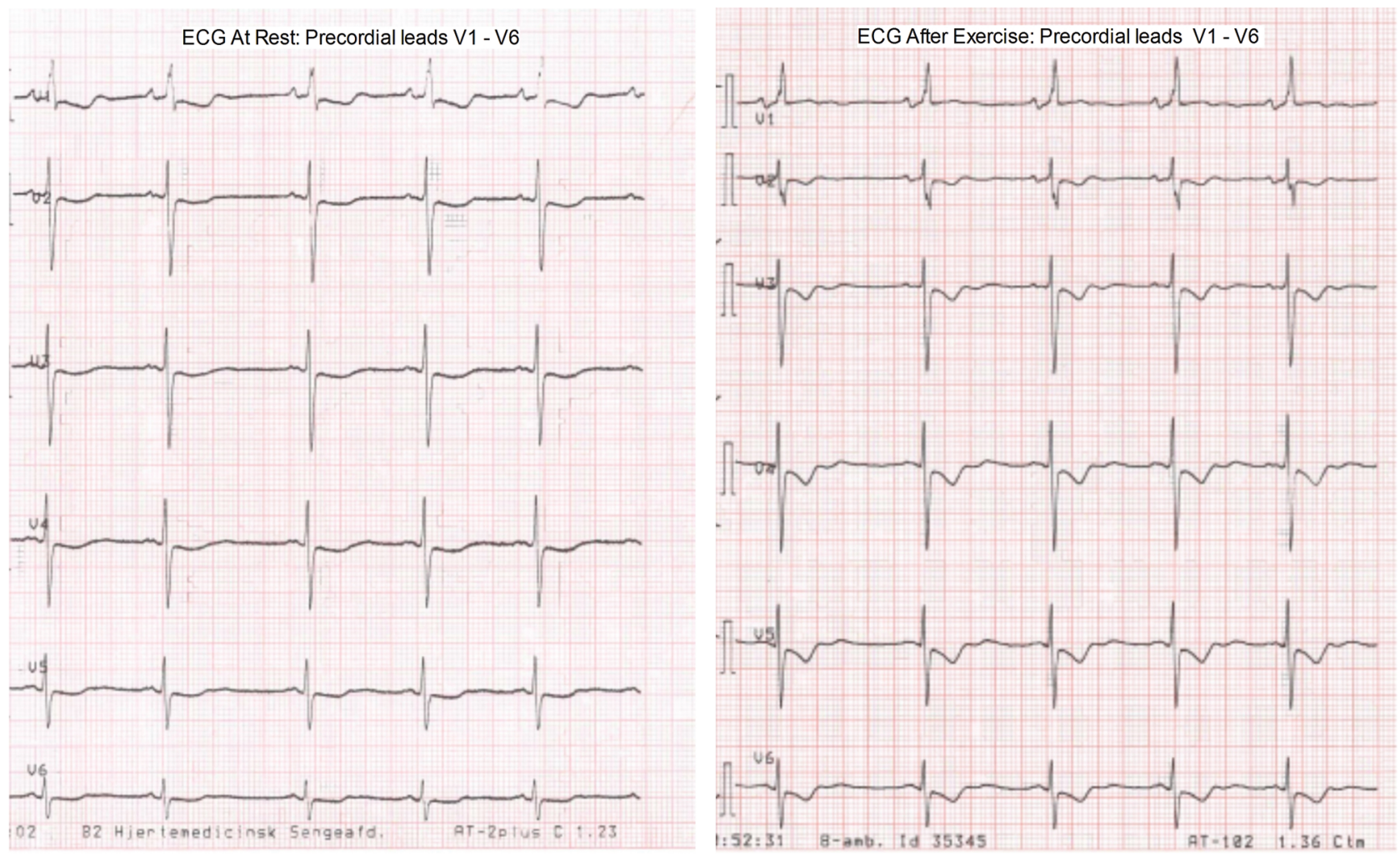

Figure 1. Twelve-lead electrocardiogram (ECG) at rest (left) and shortly after mild exercise (right). ST segment depression and T-wave inversion became pronounced during physical activity, particularly in leads $\mathrm{V}_{2}-\mathrm{V}_{6}$.

velopment of chest pain in $\mathrm{PH}$ may often be a consequence of worsening $\mathrm{PH}$ and increased RV strain, as also initially suspected in this case. However, LMCA extrinsic compression due to a dilated main PA is another very important cause of exercise-related chest pain in $\mathrm{PH}$, as finally concluded in our patient. Overlooking this mechanism of chest pain in $\mathrm{PH}$ may have serious consequences.

LMCA extrinsic compression due to a dilated PA has been reported previously. The clinical presentation may include exerciserelated chest pain, cardiogenic shock, malignant arrhythmias due to myocardial ischemia, or sudden death. ${ }^{3}$ The incidence of LMCA compression due to PA dilatation is not well known but ranges between $5 \%$ and $44 \%$, according to different case series. ${ }^{4,5}$

In contrast to atherosclerotic coronary artery disease, which is more common in elderly males, extrinsic LMCA compression appears to affect younger people and may have a higher incidence in women. ${ }^{6}$ Because of a low pretest probability for atherosclerotic coronary artery disease in young $\mathrm{PH}$ patients, coronary angiography is rarely performed. However, the extrinsic compression of the LMCA due to an enlarged PA is becoming increasingly recognizable and should always be considered in $\mathrm{PH}$ patients with exercise-induced chest pain, which is the most common clinical presentation of this problem. ${ }^{7,8}$ In one study, the origin of the LMCA from the right sinus of Valsalva was considered to convey a higher risk for extrinsic compression compared with the normal origin from the left

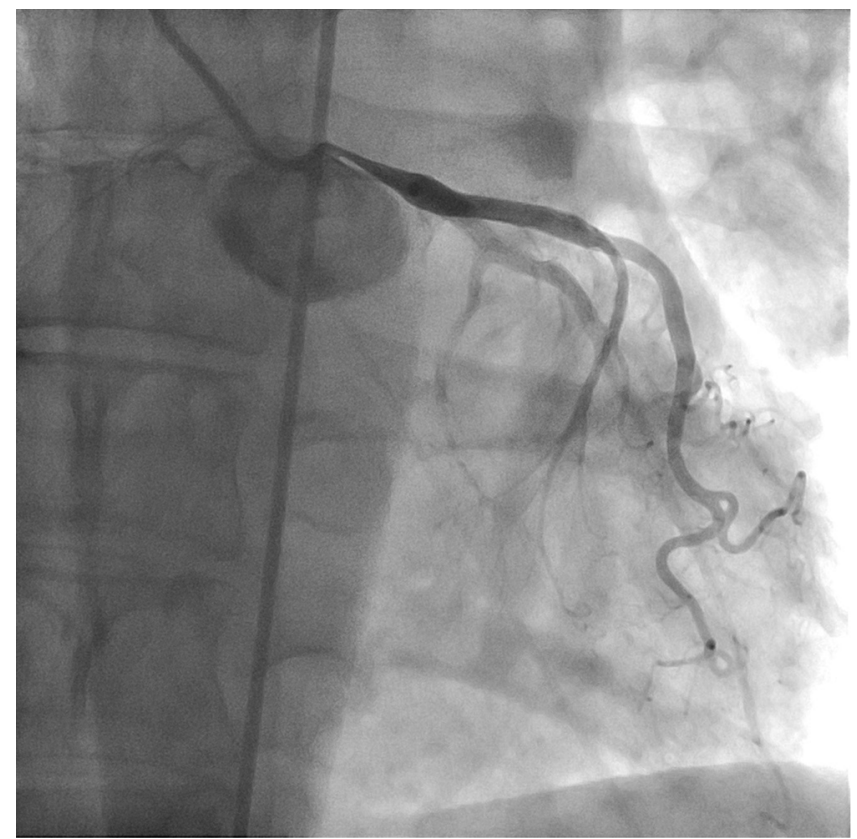

Figure 2. Coronary angiography demonstrating 90\% stenosis of the ostium of the left main coronary artery (LMCA). Additionally, highly positioned LMCA from the left sinus of the aortic root was also observed, but no other atherosclerotic changes were found. 


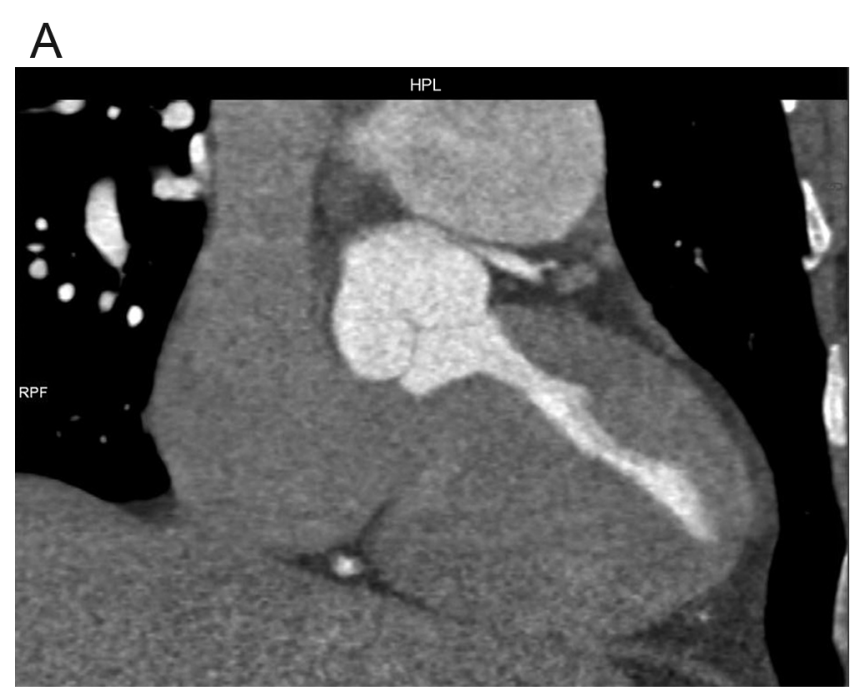

B

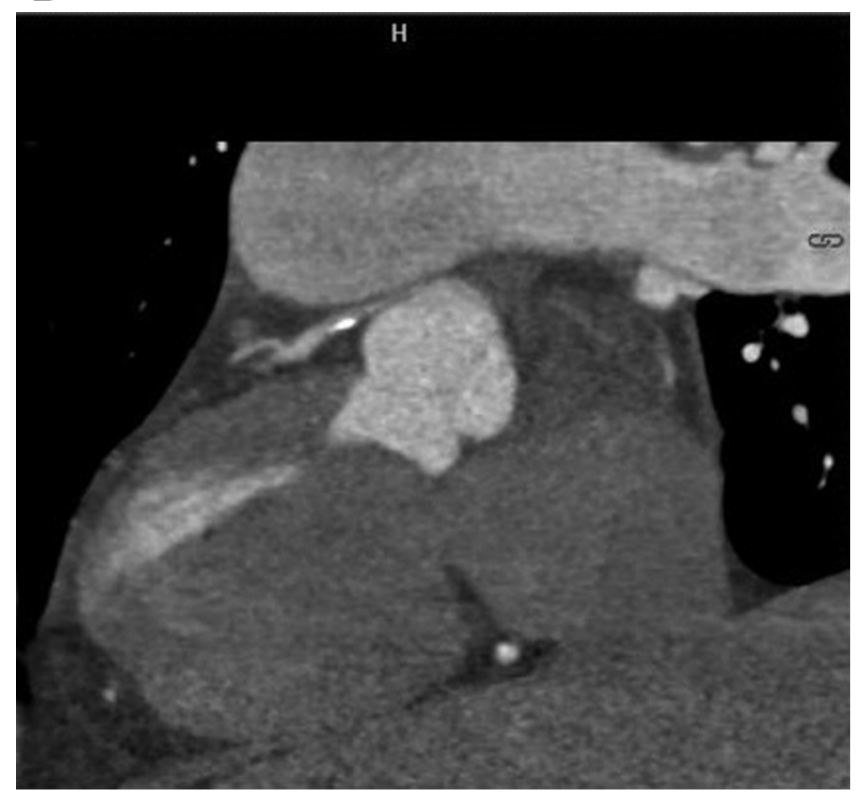

Figure 3. Computed tomography coronary angiography (A: coronal view; $B$ : sagittal view) showing left main coronary artery originating from a high position in the left coronary sinus and compressed by a dilated main pulmonary artery.

sinus. ${ }^{6}$ In another study, risk factors predisposing to LMCA compression in $\mathrm{PAH}$ were younger age, severe pulmonary trunk dilatation ( $>40 \mathrm{~mm}$; normal: $25-30 \mathrm{~mm}$ ), and a PA trunk/aorta ratio $>1.2$ (normal: 1.0$)^{5}$
Our case demonstrates that a high origin from the left coronary sinus may possibly be another risk factor for LMCA compression due to a steeper and more angled takeoff of the LMCA from the left sinus. In patients with known PAH who present with newly developed severe anginal pain during exercise, extrinsic LMCA compression from a dilated PA should be considered in the differential diagnosis. MDCT is excellent for confirming the diagnosis in such cases and may serve as the first-line diagnostic examination. Coronary angiography, IVUS, and myocardial perfusion methods to detect myocardial ischemia may play supplementary roles in the diagnostic evaluation. The treatment of LMCA extrinsic compression from a dilated PA in PAH is feasible and safe by PCI, and this has now become the most accepted treatment. $^{9}$

\section{Source of Support: Nil.}

Conflict of Interest: None declared.

\section{REFERENCES}

1. Rich S, Dantzker DR, Ayres SM, Bergofsky EH, Brundage BH, Detre KM, Fishman AP, et al. Primary pulmonary hypertension: a national prospective study. Ann Intern Med 1987;107:216-223.

2. Viar WN, Harrison TR. Chest pain in association with pulmonary hypertension: its similarity to the pain of coronary disease. Circulation 1952;5:1-11.

3. Lee MS, Oyama J, Bhatia R, Kim YH, Park SJ. Left main coronary artery compression from pulmonary artery enlargement due to pulmonary hypertension: a contemporary review and argument for percutaneous revascularization. Catheter Cardiovasc Interventions 2010;76:543-550.

4. Mitsudo K, Fujino T, Matsunaga K, Doi O, Nishihara Y, Awa J, Goto T, et al. Coronary arteriographic findings in the patients with atrial septal defect and pulmonary hypertension (ASD $+\mathrm{PH})$-compression of left main coronary artery by pulmonary trunk [in Japanese]. Kokyu To Junkan 1989;37:649-655.

5. Mesquita SM, Castro CR, Ikari NM, Oliveira SA, Lopes AA. Likelihood of left main coronary artery compression based on pulmonary trunk diameter in patients with pulmonary hypertension. Am J Med 2004;116:369-374.

6. Kajita LJ, Martinez EE, Ambrose JA, Lemos PA, Esteves A, Nogueira da Gama M, Jatene AD, Ramires JA. Extrinsic compression of the left main coronary artery by a dilated pulmonary artery: clinical, angiographic, and hemodynamic determinants. Catheter Cardiovasc Interventions 2001;52:4954

7. Kanjwal MY, Carlson DE Jr., Schwartz JS. Chronic/subacute total occlusion of the left main coronary artery-a case report and review of literature. Angiology 1999;50:937-945.

8. Shen AY, Jandhyala R, Ruel C, Lundstrom RJ, Jorgensen MB. Predictors of survival after coronary bypass grafting in patients with total occlusion of the left main coronary artery. Am J Cardiol 1998;81:343-346.

9. Lindsey JB, Brilakis ES, Banerjee S. Acute coronary syndrome due to extrinsic compression of the left main coronary artery in a patient with severe pulmonary hypertension: successful treatment with percutaneous coronary intervention. Cardiovasc Revasc Med 2008;9:47-51. 